\title{
Compressive Photon-Sieve Spectral Imaging
}

\author{
Oguzhan F. Kar ${ }^{1}$, Ulas Kamaci ${ }^{2}$, Fatih C. Akyon ${ }^{3}$, and Figen S. Oktem ${ }^{1}$ \\ ${ }^{1}$ Dept. of Electrical Eng., Middle East Technical Univ. (METU), Ankara, 06800 Turkey \\ ${ }^{2}$ Dept. of Electrical and Computer Eng., Univ. of Illinois, Urbana-Champaign, IL, 61801 USA \\ ${ }^{3}$ Dept. of Electrical Eng., Bilkent Univ., Ankara, 06800 Turkey \\ figeno@metu.edu.tr
}

\begin{abstract}
We develop a new compressive spectral imaging modality that utilizes a coded aperture and a photon-sieve for dispersion. The 3D spectral data cube is successfully reconstructed with as little as two shots using sparse recovery.(C) 2018 The Author(s)

OCIS codes: (110.1758) Computational imaging, (110.4234) Multispectral and hyperspectral imaging.
\end{abstract}

\section{Introduction}

Compressive spectral imaging has the remarkable advantage of reconstructing the entire spectral cube from a few multiplexed measurements through sparse recovery. Inspired by compressed sensing, various different optical configurations have been suggested for compressive spectral imaging. Examples include compressive coded aperture snapshot spectral imager (CASSI) [1], and compressive hyperspectral imager by separable spectral and spatial operators [2].

In this paper, we present a new compressive spectral imaging modality called compressive photon sieve spectral imaging (CPSSI). The CPSSI utilizes a coded aperture to modulate the optical field from a scene and a diffractive imaging element such as a photon-sieve [3] for dispersion. The coded field passed through the photon sieve is recorded at a few measurement planes using a moving detector. A fast sparsity-based reconstruction approach is developed to reconstruct the spectral data cube from these measurements. Simulation results show promising reconstruction performance with as little as two measurements.

\section{Forward Problem}

As illustrated in Fig. 1a, CPSSI requires a simple optical system involving (1) an objective lens, (2) a coded aperture, (3) a photon sieve as a diffractive imaging element, and (4) a monochrome detector. The objective lens forms the image of the scene on the plane of the coded aperture. The resulting coded field is passed through the photon sieve a modified Fresnel lens with the open zones replaced by circular holes. Because of the wavelength-dependent focal length of the sieve, each wavelength component is focused differently on the detector. Hence resulting measurement is a superposition of coded and differently blurred spectral bands. A total of $K$ such measurements are obtained using a moving detector, each at a different distance from the sieve.

The image formation model that relates the discretized spectral cube to the measurements can be obtained as follows:

$$
y_{k}[m, n]=\sum_{s=1}^{S}\left(c_{s}[m, n] x_{S}[m, n]\right) * h_{d_{k}, \lambda_{s}}[m, n]+w_{k}[m, n] .
$$

Here $y_{k}[m, n]$ represents the $k$ th measurement obtained at distance $d_{k}$ over $N \times N$ pixels and $w_{k}[m, n]$ accounts for the noise. The spectral cube is discretized into $S$ spectral bands, and $x_{s}[m, n]$ denotes the intensity of the sth discrete band with wavelength $\lambda_{s}$. This spectral band is modulated with the coded aperture $c_{S}[m, n]$, as differently from the non-compressive setting [3], and then convolved with the PSF, $h_{d_{k}, \lambda_{s}}[m, n]$, of the photon sieve at distance $d_{k}$. The closed-form expression for this PSF is given elsewhere [3,4]. If a traditional block-unblock (i.e. uncolored) mask is used, $c_{s}[m, n]$ is same for all spectral bands $(s=1, \ldots, S)$; however, this is not the case if a colored coded aperture [5] is used instead, since in this case different wavelengths are modulated differently.

Using lexicographic ordering, the above image-formation model can be cast in matrix-vector form as follows:

$$
y=\left[\begin{array}{c}
y_{1} \\
\vdots \\
y_{K}
\end{array}\right], x=\left[\begin{array}{c}
x_{1} \\
\vdots \\
x_{S}
\end{array}\right], H=\left[\begin{array}{ccc}
H_{1,1} & \ldots & H_{1, S} \\
\vdots & & \vdots \\
H_{K, 1} & \ldots & H_{K, S}
\end{array}\right], C=\left[\begin{array}{ccc}
\operatorname{diag}\left(c_{1}\right) & \ldots & 0 \\
\vdots & \ddots & \vdots \\
0 & \ldots & \operatorname{diag}\left(c_{S}\right)
\end{array}\right] .
$$

Here $y$ is the overall measurement vector containing all the $K$ measurements, and $x$ is the complete data cube in vector form. The $N^{2} \times N^{2}$ matrix $H_{k, s}$ represents the convolution operation with $h_{d_{k}, \lambda_{s}}$, and $H$ is the overall sensing matrix of size $K N^{2} \times S N^{2}$. The diagonal matrix $C$ corresponds to the coding operation, and takes values 1 or 0 along its diagonal. 

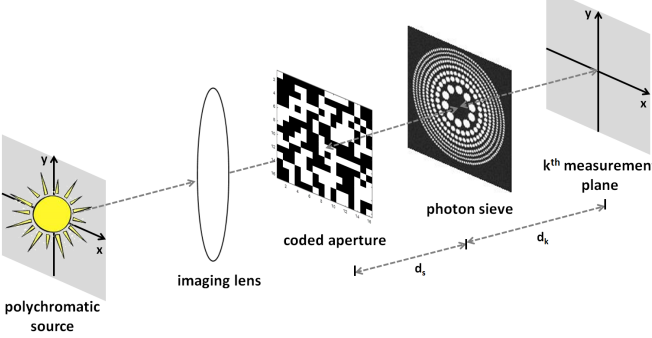

(a)

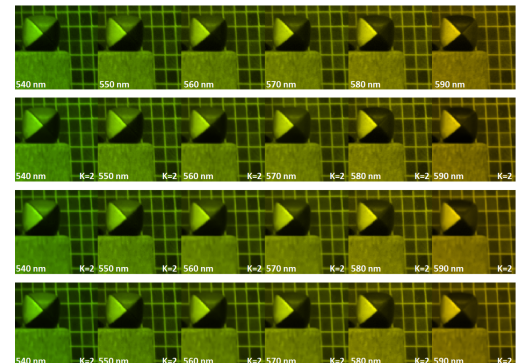

(b)

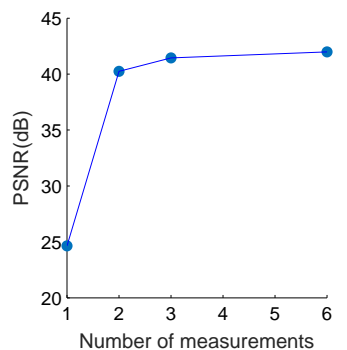

(c)

Fig. 1: (a) CPSSI system. (b) Reconstructed images from compressive measurements $(K=2)$. Top to bottom: true images, reconstructions with no mask, uncolored mask, colored mask. (c) PSNR versus $K$ for the colored mask case

\section{Image Reconstruction Approach}

In the inverse problem, the goal is to recover the unknown spectral images, $x$, from their noisy, superimposed, coded and blurred measurements, $y$. To enforce sparsity, this underdetermined problem is formulated as follows:

$$
\min _{x, b} v\|y-H C x\|^{2}+\|W x-b\|^{2} \text { s.t. }\|b\|_{0} \leq \beta
$$

Here $W$ represents the patch-based 3D DCT operator, and $b$ is the corresponding sparse code vector. To solve this optimization problem, we develop a fast alternating minimization approach which alternately minimizes the objective term in Eqn. (3) over the spectral images $x$ and sparse codes $b$. In the sparse coding step, the objective is minimized over $b$ while $x$ is kept fixed; the optimal solution is obtained by assigning the largest $\beta$ elements of $W x$ to $b$, and zeroing the others. In the spectral cube update step, the minimization problem over $x$ is solved while $b$ is kept fixed. This is a least-squares problem whose linear normal equations, in the form of $A x=t$ with $A=v C H^{*} H C+W^{*} W$ and $t=v C H^{*} y+W^{*} b$, are solved efficiently using a conjugate-gradient algorithm.

\section{Numerical Results}

Here we present numerical simulations to illustrate the performance of the proposed imaging technique. We consider a dataset of size $128 \times 128 \times 6$ ( 6 wavelengths from $540-590 \mathrm{~nm}$ with $10 \mathrm{~nm}$ spacing), which was obtained from an online hyperspectral image database at the University of Manchester. For the photon sieve, the outer diameter is selected as $3.36 \mathrm{~mm}$ and the diameter of the smallest hole as $15 \mu \mathrm{m}$, resulting in a focal length of $9 \mathrm{~cm}$ at $560 \mathrm{~nm}$. The measurements are simulated using the model in (2) with randomly generated colored or uncolored coded masks.

In the first analysis, 2 noiseless measurements are taken at the focal planes of the second and fifth spectral components by using no mask, uncolored and colored coded apertures. Reconstructed images for these three cases are given in Fig. $1 \mathrm{~b}$ together with the true images. The results demonstrate successful reconstruction of the spectral cube from compressive measurements with a PSNR of more than $40 \mathrm{~dB}$ when either uncolored or colored masks are used. For the second analysis, the above experiment is repeated for $K=1,3$, and 6 cases, with the 3 measurements taken at the midpoints of successive wavelength sources in the two extreme ends, and one in the center. Fig. 1c shows the reconstruction performance for different compression ratios for the colored mask case. This suggests that compression ratios of up to $33 \%$ allows almost perfect recovery. In the third analysis, the effect of noise is explored by generating noisy measurements with different SNRs. Table 1 shows the reconstruction PSNRs for the $K=2$ and $K=3$ cases with a colored mask, and demonstrates that the reconstruction performance degrades gracefully at noisy regimes.

In conclusion, the developed compressive spectral imaging modality, which relies on a novel and simple optical configuration with one diffractive imaging element, offers promising performance with as little as two shots. The performance can be improved further with the optimization of coded apertures and use of adaptive sparsity priors.

Table 1: Comparison of average PSNRs (dB) for different compressive measurement scenarios and SNRs.

\begin{tabular}{|c|c|c|}
\hline SNR (dB) & $\mathbf{K = 2}$ (33\% compression) & $\mathbf{K = 3}$ (50\% compression) \\
\hline 20 & 30.69 & 33.97 \\
\hline 30 & 32.92 & 34.98 \\
\hline Infinite & 40.25 & 44.45 \\
\hline
\end{tabular}

\section{References}

1. G. Arce et al., “Compressive coded aperture spectral imaging: An introduction,” IEEE Sig. Process. Mag., 31, 105-115 (2014).

2. Y. August et al., "Compressive hyperspectral imaging by random separable projections" Applied optics, 52, D46-D54 (2013).

3. F. S. Oktem et al., "High-resolution computational spectral imaging with photon sieves," in 2014 IEEE ICIP Conf., pp. 5122-5126.

4. F. S. Oktem et al., "Image formation model for photon sieves," in 2013 IEEE ICIP Conf., pp. 2373-2377.

5. H. Arguello and G. R. Arce, "Colored coded aperture design by concentration of measure in compressive spectral imaging," IEEE Trans. Image Process. 23, 1896-1908 (2014). 